\title{
Determinación de áreas de recarga hídrica en las subcuencas de los ríos Frijoles y Guacalito, Upala, Costa Rica
}

\author{
Luis Diego Mora-Jiménez ${ }^{1}$ \& Jeffrey Ruiz- Valverde ${ }^{2}$ \\ 1. M.Sc. Universidad Estatal a Distancia, Costa Rica. Escuela de Ciencias Exactas y Naturales. Cátedra de Sociedad y \\ Ambiente. Profesor tutor. Correo: Imoraj@uned.ac.cr \\ 2. Lic. Hidroeléctrica Los Negros I y II, Servicios Públicos de Heredia. Gestor Ambiental. Correo: jruizv@Esph-sa.com
}

Recibido: 10 de octubre de 2017

Aceptado: 11 de febrero de 2018

\begin{abstract}
RESUMEN
El estudio se realizó en la parte media y alta de las subcuencas Frijoles y Guacalito, localizadas en el sector norte del volcán Miravalles, pertenecientes al distrito Aguas Claras y Upala respectivamente, del cantón Upala, provincia Alajuela. El objetivo principal fue determinar áreas potenciales de recarga hídrica en la parte media y alta de las subcuencas Frijoles y Guacalito a través de la integración de variables biofísicas. La investigación se desarrolló en tres etapas: la primera, corresponde a la caracterización biofísica de la parte media y alta de las subcuencas Frijoles y Guacalito; la segunda, definir puntos de muestreo con mayor posibilidad de recarga basado en los criterios de pendiente y uso de suelo y; tercero, utilizar el método llamado balance hídrico de suelos (Schosinsky, 2006), el cual permitió estimar la recarga potencial en los puntos de muestreos seleccionados con el fin de proponer lineamientos de protección de acuerdo con la condición de uso de la tierra en las áreas de recarga hídrica.
\end{abstract}

Palabras claves: Área de recarga hídrica, infiltración, recarga potencial, balance hídrico de suelos.

\begin{abstract}
The study was carried out in the middle and upper part of the Frijoles and Guacalito sub-basins, located in the northern sector of the Miravalles volcano, belonging to the Aguas Claras and Upala districts, respectively, of the Upala canton, Alajuela province. The main objective was to determine potential areas of water recharge in the middle and upper part of the Frijoles and Guacalito subbasins through the integration of biophysical variables. The research was developed in three stages: the first, corresponds to the biophysical characterization of the middle and upper part of the sub-basins Frijoles and Guacalito; the second, define sampling points with a greater possibility of recharge based on the criteria of slope and land use; third, to use the so-called soil water balance method (Schosinsky,
\end{abstract}

2006), which allowed estimating the potential recharge at the selected sampling points in order to propose protection guidelines according to the condition of land use in the areas of water recharge.

Key words: Water recharge area, infiltration, potential recharge, water balance of soils.

\section{INTRODUCCIÓN}

Los sitios donde el agua, producto de la infiltración, recargan los acuíferos se conocen como áreas de recarga hídrica. Estás áreas son definidas por la Ley forestal (1996) como superficies cuya penetración del agua alimenta los acuíferos y los cauces de los ríos.

El Centro Agronómico Tropical de Investigación y Enseñanza (CATIE) ha apoyado investigaciones dirigidas hacia la identificación de áreas de recarga hídrica en Costa Rica y en el resto de Centroamérica, a partir de la metodología del balance hídrico de Schosinsky Nevermann (2006). Por ejemplo, Noriega Arriaga (2005) aplicó la metodología en la cuenca del río Sibacá, Guatemala, para estimar las áreas potenciales de recarga hídrica, o la investigación desarrollada por Blanco Rojas (2010), utilizando la metodología para estimar zonas de infiltración en la parte alta de tres microcuencas de la zona norte de Costa Rica.

La regulación de áreas importantes de recarga hídrica está tipificado explícitamente en la legislación costarricense a través de la Política hídrica nacional, la Ley de Aguas No. 276 en su 
Artículo 31 declarando como reserva de dominio a favor de la Nación la zona forestal que protege los terrenos donde se produce la infiltración del agua, así mismo, la Ley Forestal en su Artículo 33 definiendo como áreas de protección las zonas de recarga y los acuíferos de los manantiales.

Costa Rica, a pesar de ello, no ha establecido una meta clara para la identificación de estas áreas prioritarias; por el contrario, cada día las fuentes de abastecimiento se ven contaminadas por prácticas agrícolas y ganaderas inadecuadas en sitios próximos a la insurgencia de manantiales, limitando la calidad y cantidad del recurso hídrico.

Por tal razón, el objetivo principal fue determinar áreas potenciales de recarga hídrica en la parte media y alta de las subcuencas Frijoles y Guacalito a través de la integración de variables biofísicas (precipitación, evapotranspiración potencial, cobertura vegetal, rango de pendiente, velocidad de infiltración, profundidad de las raíces, intercepción de lluvia en el follaje y humedad inicial del suelo), lo cual contribuirá al manejo y conservación de estas áreas.

\section{MATERIALES Y MÉTODOS}

El área de estudio se localiza en las coordenadas planas (CRTM05) 370909 Este, 1197362 Norte y 300248 Este, 1188888 Norte en la hoja topográfica Miravalles a escala 1:50000. Se ubica en la parte norte del volcán Miravalles y comprende la parte media y alta de las subcuencas Frijoles y Guacalito con un área de $42 \mathrm{~km}^{2}$ (Figura 1).

La zona de interés presenta un clima tropical húmedo con un promedio anual de precipitación de $4000 \mathrm{~mm}$. La temperatura tienen un promedio de $24,5^{\circ} \mathrm{C}$ con una oscilación térmica que varía entre los 23 y los $26{ }^{\circ} \mathrm{C}$ (Instituto Meteorológico Nacional, 2012). En la zona se identifican relieves multiconvexos, que actúan de manera diferente en el sistema hídrico de las subcuencas. La mayor altitud en la zona alcanza los 2028 m.s.n.m. en la cúspide del volcán Miravalles.

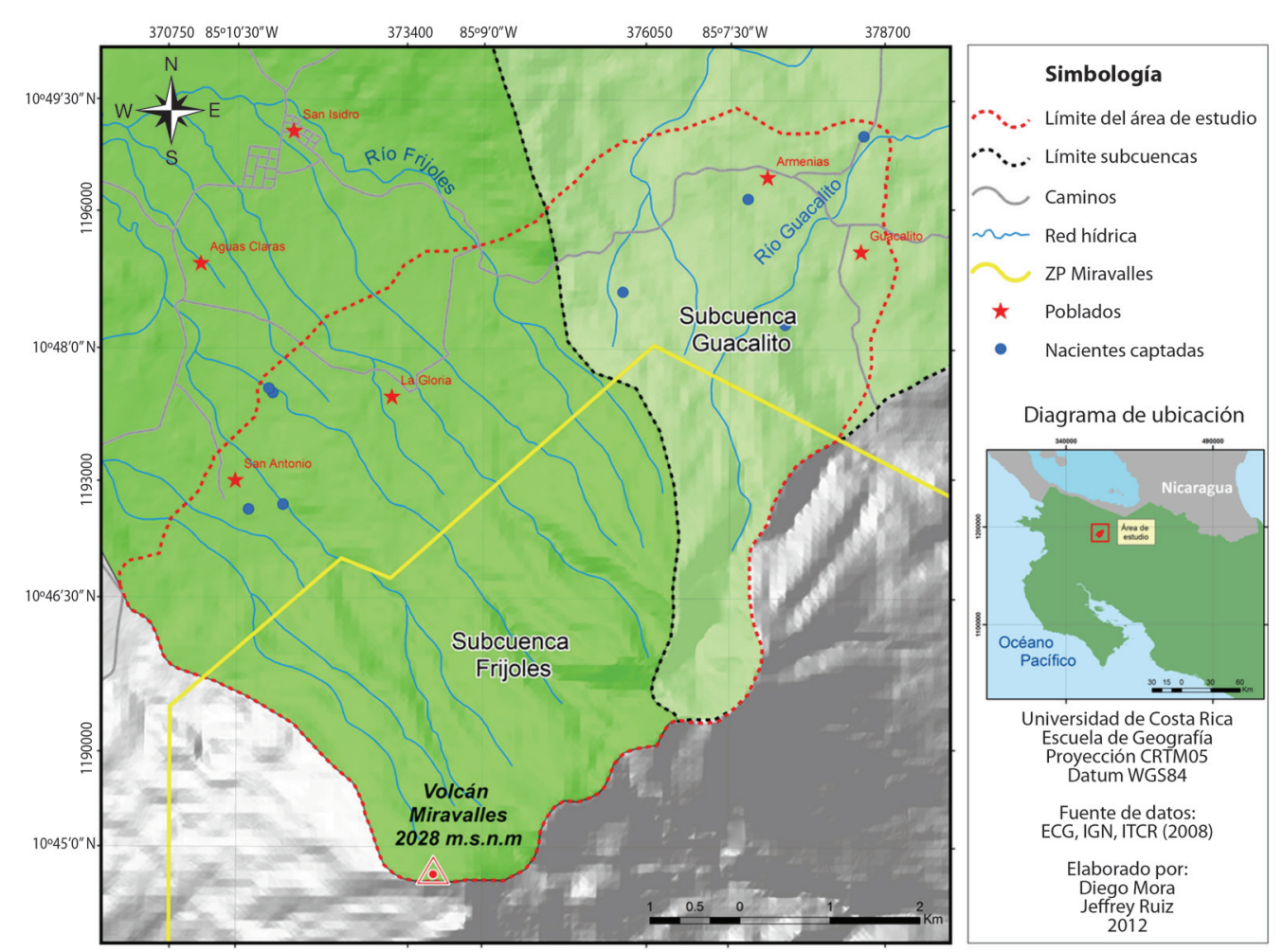

Figura 1. Localización del área de estudio, parte media y alta de las subcuencas Frijoles y Guacalito, Upala. 

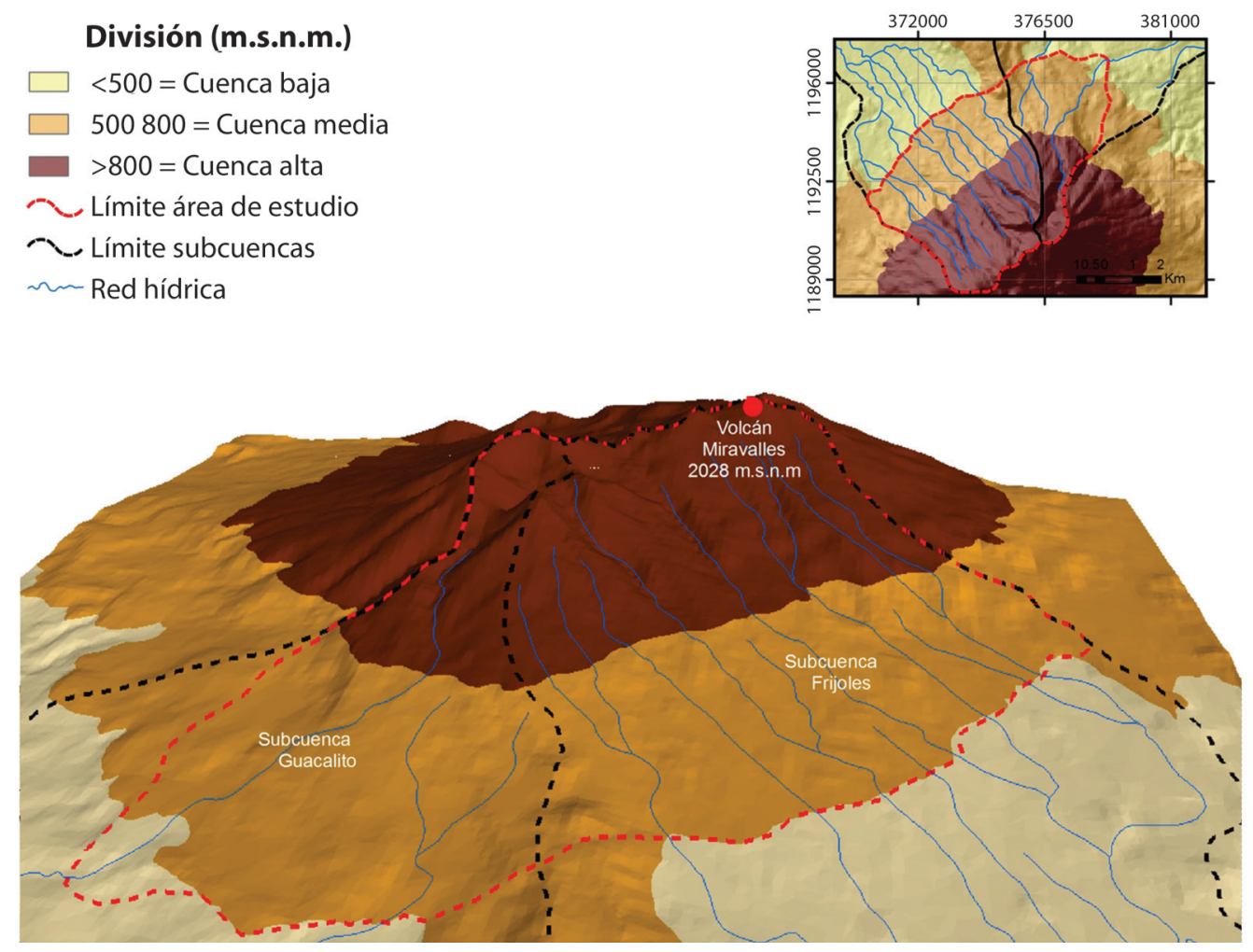

Figura 2. División estructural de la subcuenca Frijoles y Guacalito, Upala. Elaborado por Mora y Ruiz, (2012).

Inicialmente, la delimitación del área de estudio se realizó con un sistema de información geográfico para definir la parte media y alta de las subcuencas Frijoles y Guacalito (Figura 2). Posteriormente, se establecieron puntos de muestreo basado en los criterios de pendiente y uso de suelo. En cada punto de muestreo, se procedió a la recolección y análisis de información de distintas variables biofísicas, para su posterior integración en una base de datos.

Posteriormente, el cálculo de recarga potencial de acuíferos mediante un balance hídrico de suelos consistió en la determinación de las siguientes variables biofísicas:

1. Precipitación y Evapotranspiración potencial (ETP).

2. Suelo: se recolectaron 90 muestras de las cuales; 30 se utilizaron para determinar la textura, 30 para determinar la densidad aparente y las restantes, para determinar la capacidad de campo.
3. Análisis de laboratorio: Las muestras recolectadas fueron pesadas con su humedad de campo, con la finalidad de determinar el porcentaje de humedad presente en el suelo en el momento de extracción de la muestra. La textura, capacidad de campo y densidad aparente, se calcularon siguiendo los pasos explicados en la Guía práctica para el estudio introductorio de los suelos con un enfoque agrícola de Henríquez y Cabalceta (1999).

4. Uso actual de la tierra: Se determinó a través de imágenes satelitales, verificación en campo y el empleo de sistemas de información geográfica. A cada uso se le ajustó un coeficiente $(\mathrm{Kv})$ establecido por Schosinsky Nevermann (2006)mostrada en la tabla 1.

5. Pendientes: se elaboró un mapa digital de pendientes en porcentajes a partir de curvas de nivel cada 20 metros. Los rangos y los coeficientes utilizados se muestran en la tabla 2.

6. Infiltración básica: Se calculó a través del método llamado infiltrómetro de doble 
TABLA 1

\section{Coeficiente de cobertura vegetal (Kv)} según el uso de la tierra

\begin{tabular}{lc}
\multicolumn{1}{c}{ Cobertura vegetal de Schosinsky } & $\mathrm{Kv}$ \\
Bosques & 0,2 \\
Cultivos & 0,1 \\
Zacate $75 \%$ & 0,21 \\
Cobertura con pastizal & 0,18 \\
Pastos con árboles dispersos & 0,18 \\
\hline
\end{tabular}

Elaborado por Mora y Ruiz, (2012). Fuente: Schosinsky, (2006).

TABLA 2

Coeficiente de pendientes $(\mathrm{Kp})$ según los rangos de pendiente

\begin{tabular}{cccc} 
No. & Terreno & $\begin{array}{c}\text { Rangos de } \\
\text { pendiente en } \%\end{array}$ & $\mathrm{kp}$ \\
\hline 1 & Muy regular & $0-0,20$ & 0,3 \\
2 & regular & $0,21-1$ & 0,2 \\
3 & Algo regular & $1,1-2$ & 0,15 \\
4 & Poco regular & $2,1-7$ & 0,1 \\
5 & Muy irregular & Mayor de 7 & 0,06 \\
\hline
\end{tabular}

Elaborado por Mora y Ruiz, (2012). Fuente: Schosinsky (2006).

TABLA 3

Velocidades y Clases de infiltración

\begin{tabular}{cc}
$\begin{array}{c}\text { Velocidad de infiltración } \\
\text { (cm por hora) }\end{array}$ & Clases de infiltración \\
$>50,8$ & Muy rápido \\
$15,24-50,80$ & Rápido \\
$50,80-15,24$ & Moderadamente rápido \\
$15,24-5,08$ & Moderado \\
$5,08-1,52$ & Moderadamente lento \\
$1,52-0,51$ & Lento \\
$0,51-0,0038$ & Muy lento \\
$<0,0038$ & Impermeable \\
\hline
\end{tabular}

anillo. Consistió en instalar en el terreno, previamente limpio de hierbas, piedras, etc., dos cilindros concéntricos: uno interno de $30 \mathrm{~cm}$ de diámetro y $30 \mathrm{~cm}$ de altura y uno externo de $50 \mathrm{~cm}$ de diámetro con una altura de $20 \mathrm{~cm}$. La interpretación de los datos de velocidades de infiltración se dio por medio de una clasificación de velocidades propuesta por el Departamento de Agricultura de los Estados Unidos (1999), la cual mide que tan rápido o lento puede infiltrarse el agua en el suelo. La tabla 3 muestra las velocidades de infiltración y su clasificación de acuerdo con las propiedades del suelo referidas a una velocidad de infiltración estable.

7. Profundidad de las raíces: Los valores de profundidad de las raíces extractoras de agua, según el tipo de cobertura vegetal del suelo, fueron determinadas, por medio de observación en los cortes de camino para, posteriormente, ser medidas y comparadas con los valores sugeridos por Schosinsky Nevermann (2006) en la metodología del balance hídrico de suelos. Para determinar la humedad que extraen las raíces de cada cobertura fue necesario convertir el porcentaje de humedad por volumen en $\mathrm{mm}$ de agua existentes en la franja de profundidad de las raíces resolviendo la siguiente ecuación:

$$
\begin{gathered}
\mathrm{mm} \text { de agua }=(\text { porcentaje de humedad por } \\
\text { volumen })^{*}(\text { profundidad de las raíces en } \mathrm{mm})
\end{gathered}
$$

8. Determinar la intercepción de la lluvia en el follaje. Los coeficientes referidos a la intercepción de la lluvia en el follaje según el tipo de cobertura vegetal del suelo, fueron determinados en la tabla 4 previamente establecidos por Schosinsky.

TABLA 4

\section{Coeficiente de retención de lluvia en el follaje según tipo de cobertura vegetal del suelo}

\begin{tabular}{lcc}
\multicolumn{1}{c}{ Tipo de cobertura vegetal del suelo } & Retención de lluvia en el follaje (\%) & Retención de lluvia en el follaje (100\%=1\%) \\
$\begin{array}{l}\text { General (cualquiera que } \\
\text { no sea bosque denso) }\end{array}$ & 12 & 0,12 \\
$\begin{array}{l}\text { Bosque denso } \\
\text { B }\end{array}$ & 20 & 0,2 \\
\hline
\end{tabular}

Elaborado por Mora y Ruiz, 2012. Fuente: Schosinsky (2006) 
9. Humedad inicial del suelo: La humedad inicial es considerada como la capacidad de campo (CC) en milímetros obtenida para cada tipo de suelo y cobertura en los meses de enero y febrero cuando los suelos aún mantenían su humedad producto de las lluvias de los meses anteriores. Se aplicó la siguiente fórmula para obtener la CC en milímetros.

$$
\mathrm{CC} \mathrm{mm}=\mathrm{CC} \% / 100 / \mathrm{Da} \mathrm{g} / \mathrm{cm}^{3 *} \mathrm{PR} \mathrm{mm}
$$

Donde (CC) es la capacidad de campo; (Da) es la densidad aparente y (PR) es la profundidad de las raíces

10. Determinación y clasificación de la recarga hídrica potencial del acuífero ( $R p)$ : La información generada en los pasos anteriores, fue la base para realizar la ecuación general del balance hídrico de suelos. Esta ecuación permitió determinar la cantidad potencial de agua que infiltra en los distintos puntos de muestreo (Rp). Los resultados de la recarga potencial fueron interpolados para todo el área de estudio y posteriormente fueron reclasificados según el potencial de recarga y extensión.

11. Determinación del volumen de recarga acuífera: El volumen de agua que recarga al acuífero en cada área de recarga hídrica (ARH), se determinó mediante la siguiente ecuación (Schosinsky Nevermann, 2006):

$$
\mathrm{V}=(\mathrm{Rp})^{*}(\mathrm{~A})
$$

Donde; (V) es el volumen de recarga en / año; (Rp) la recarga potencial al acuífero en m/año y (A) el área donde se genera la recarga potencial en $m^{2}$.

El objetivo de determinar el volumen de recarga, radica en cuantificar la cantidad de agua que ingresa en cada área de recarga hídrica. Los resultados obtenidos se agruparon en áreas de recarga hídrica (ARH) bajo tres categorías según el volumen: alta, media y baja.

La distribución de las categorías se realizó por medio de un sistema de información geográfico (SIG), en el cual se creó un mapa que muestra la categoría a la que pertenece cada ARH según el volumen de recarga potencial (Rp). Esta categorización ayudó a una mejor interpretación y análisis de cada área de recarga hídrica.

Finalmente, la condición de uso en cada ARH se determinó sobreponiendo las capas de uso de la tierra y capacidad de uso, en donde se utilizó un cuadro matriz de análisis de tipo cualitativo del MINAE-MAG (2001) en donde se indicaron las categorías del cruce de cada una de estas dos variables

\section{RESULTADOS}

Los resultados obtenidos de Rp, por medio del balance hídrico de suelos, se muestran en la Figura 3, el cual divide la zona de estudio en áreas $\left(\mathrm{km}^{2}\right)$ según su recarga potencial.

La distribución de las áreas de recarga hídrica se han dividido en siete áreas de acuerdo con el potencial de recarga, siendo el ARH 1 la que presenta una mayor Rp anual, mientras que la ARH7, la de menor Rp anual. De igual manera, el ARH3 abarca mayor cantidad de área en el área de estudio, mientras que el ARH7 es la que abarca menor cantidad de área (tabla 5).

La primera (ARH 1) corresponde a un área de $6 \mathrm{~km}^{2}$ del total del área de estudio $\left(42 \mathrm{~km}^{2}\right)$ y presenta una recarga potencial entre los 2253 a $2540 \mathrm{~mm} / \mathrm{año}$, se localiza principalmente en la parte alta de la subcuenca Frijoles a partir de los 800 m.s.n.m. Es el área con los mayores

\section{TABLA 5}

\section{Código y nombre de las áreas potenciales de recarga hídrica}

\begin{tabular}{ccc} 
Código & Nombre & Área $\left(\mathrm{Km}^{2}\right)$ \\
ARH1 & Área de recarga hídrica 1 & 6,36 \\
ARH2 & Área de recarga hídrica 2 & 11,51 \\
ARH3 & Área de recarga hídrica 3 & 12,31 \\
ARH4 & Área de recarga hídrica 4 & 5,24 \\
ARH5 & Área de recarga hídrica 5 & 3,56 \\
ARH6 & Área de recarga hídrica 6 & 2,74 \\
ARH7 & Área de recarga hídrica 7 & 1,04 \\
Total & & $\mathbf{4 2 , 7 6}$ \\
\hline
\end{tabular}

Elaborado por Mora y Ruiz, (2012). 

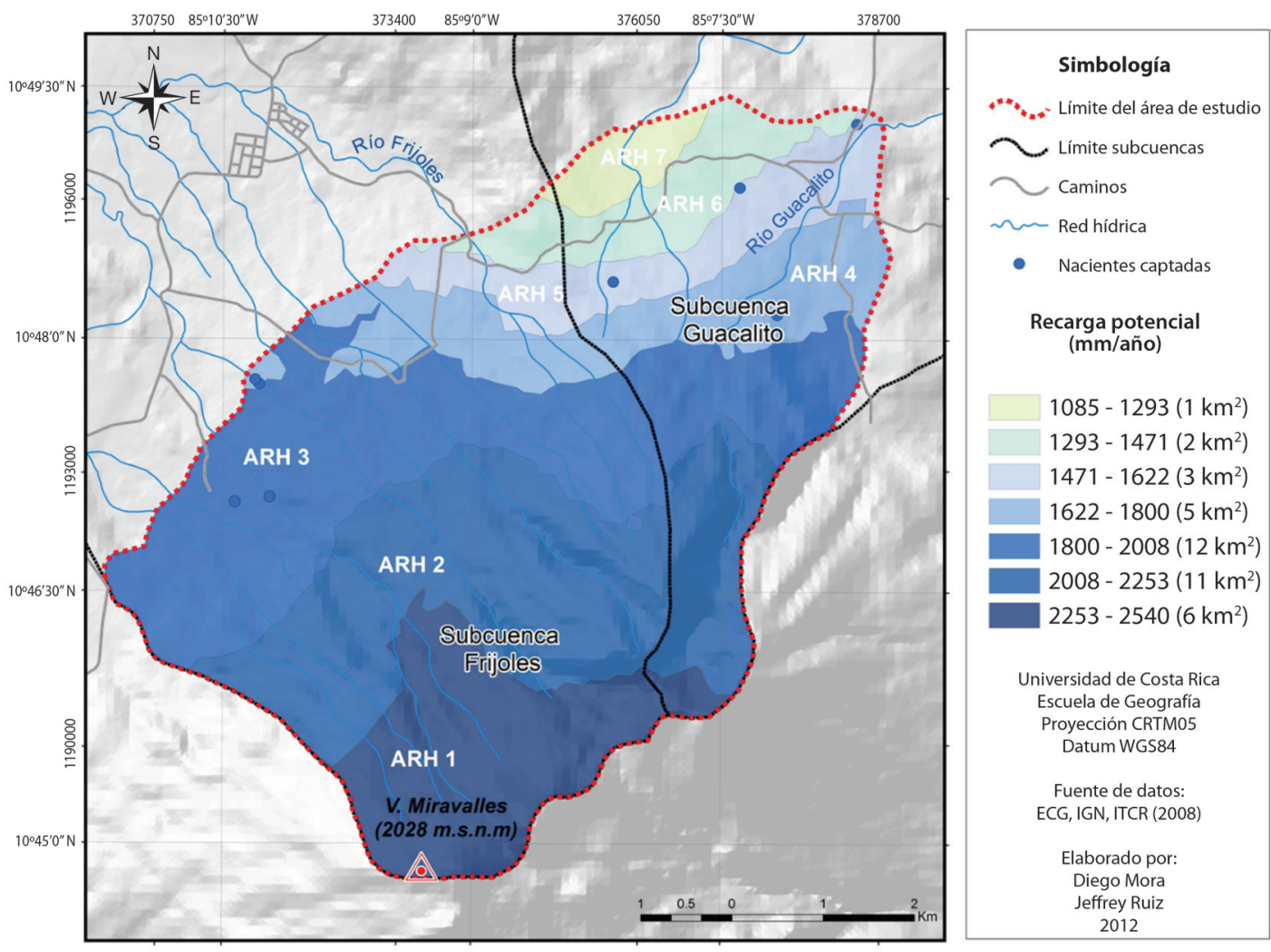

Figura 3. Áreas de recarga hídrica (ARH) en la parte media y alta de las subcuencas Frijoles y Guacalito, Upala.

rangos de recarga potencial cubierta totalmente de bosque y cuyas pendientes alcanzan sus máximos rangos.

En el área de estudio, aproximadamente 10 millones de metros cúbicos de agua al año podrían ser parte del agua subterránea, lo que refleja que en la parte media y alta de las subcuencas Frijoles y Guacalito, existen características biofísicas y fisiográficas que favorecen las recarga hídrica. La tabla 6 muestra la forma en que está distribuido el volumen anual de Rp en cada área de recarga.

La distribución del volumen de Rp en cada área de recarga hídrica, presenta diferencias debido a la heterogeneidad de las variables fisiográficas y biofísicas que interactúan en el área de

TABLA 6

Volumen de las áreas potenciales de recarga hídrica (ARH)

\begin{tabular}{cccc} 
ARH & Área $\left(\mathrm{Km}^{2}\right)$ & Volumen de Rp $\left(\mathrm{m}^{3} / \mathrm{año}\right)$ & Volumen de Rp (\%) \\
1 & 6,36 & 1824753,75 & 19,46 \\
2 & $11 ., 51$ & 2814801,89 & 30,02 \\
3 & 12,31 & 2565698,38 & 27,36 \\
4 & 5,24 & 929982,83 & 9,92 \\
5 & 3,56 & 538432,54 & 5,74 \\
6 & 2,74 & 486545,83 & 5,19 \\
7 & 1,04 & 217444,22 & 2,32 \\
Total & $\mathbf{4 2 , 7 6}$ & $\mathbf{9 3 7 7 6 5 9 , 4 3}$ & $\mathbf{1 0 0}$ \\
\hline
\end{tabular}

Elaborado por Mora y Ruiz, (2012). 
TABLA 7

Categorización de las ARH según el volumen de Rp

\begin{tabular}{cccc} 
ARH & Área $\left(\mathrm{Km}^{2}\right)$ & Volumen de $\mathrm{Rp}\left(\mathrm{m}^{3} / \mathrm{año}\right)$ & Categoría \\
1 & 6,36 & 1824753,75 & Media \\
2 & 11.51 & 2814801,89 & Alta \\
3 & 12,31 & 2565698,38 & Alta \\
4 & 5,24 & 929982,83 & Media \\
5 & 3,56 & 538432,54 & Baja \\
6 & 2,74 & 486545,83 & Baja \\
7 & 1,04 & 217444,22 & Baja \\
Total & $\mathbf{4 2 , 7 6}$ & $\mathbf{9 3 7 7 6 5 9 , 4 3}$ & \\
\hline
\end{tabular}

Elaborado por Mora y Ruiz, (2012).

TABLA 8

Volumen de Rp y extensión de las categorías a la que pertenece cada ARH

\begin{tabular}{lcccc}
\multicolumn{1}{c}{ Categoría } & \multicolumn{2}{c}{ Volumen de recarga hídrica potencial } & \multicolumn{2}{c}{ Extensión } \\
\hline Alta & $\mathrm{m}^{3} / \mathrm{año}$ & $\%$ & $\mathrm{Km}^{2}$ & $\%$ \\
Media & 5380500,27 & 57,38 & 17,87 & 55,71 \\
Baja & 2754736,58 & 29,38 & 11,60 & 27,12 \\
Total & 1242422,58 & 13,25 & 11,53 & 17,17 \\
\hline
\end{tabular}

Elaborado por Mora y Ruiz, (2012).

estudio. La clasificación del volumen permite la definición de categorías de recarga las cuales se detallan en la tabla 7.

La distribución de las categorías del volumen de Rp (Figura 4) muestra que las ARH con alto volumen de recarga potencial cubren sectores boscosos y también sectores de pastos en la parte media de ambas subcuencas. La tabla 8 detalla que casi un 55\% del área de estudio presenta áreas de recarga hídrica con alto potencial, las cuales representan casi un $57 \%$ del total del volumen de recarga hídrica. La extensión de áreas con un Rp alto presentan una proporción importante en comparación con las ARH de recarga media, que representan casi un $27 \%$ del total del área de estudio con un volumen de recarga de $29 \%$, y que las ARH de recarga baja que tan sólo representan un $17 \%$ del total del área con un volumen de recarga de apenas el $13 \%$.

La condición de uso en el área de estudio, presenta cinco categorías producto de la superposición de las capas cartográficas del uso de la tierra y la capacidad de uso. La figura 5 muestra el porcentaje y área de ocupación de las categorías de condición de uso.

Las tierras bien utilizadas hacen referencia a que, de acuerdo con las propiedades físicas (suelo, pendiente, profundidad del suelo, pedregosidad, clima, entre otros) las actividades agropecuarias que se vienen desarrollando en dichas áreas, son compatibles con las características mencionadas y por lo tanto no corren el riesgo de degradar los suelos. En la zona de estudio, está categoría representa la mayor extensión territorial abarcando un espacio de $21,7 \mathrm{~km}^{2}$ que corresponden a un 50\% del total del área de estudio. Se localizan, principalmente, en la parte alta de ambas subcuencas aunque también se encuentran en algunos parches de bosque a lo largo de ríos y quebradas de las subcuencas.

Por el contrario, Las tierras gravemente sobreutilizadas son aquellas que están sumamente erosionadas, degradadas y que necesitan con urgencia una intervención en el manejo que se les da. Su distribución en el área de estudio es de $1,9 \mathrm{~km}^{2}$ el cual corresponde a un $4 \%$ del total 

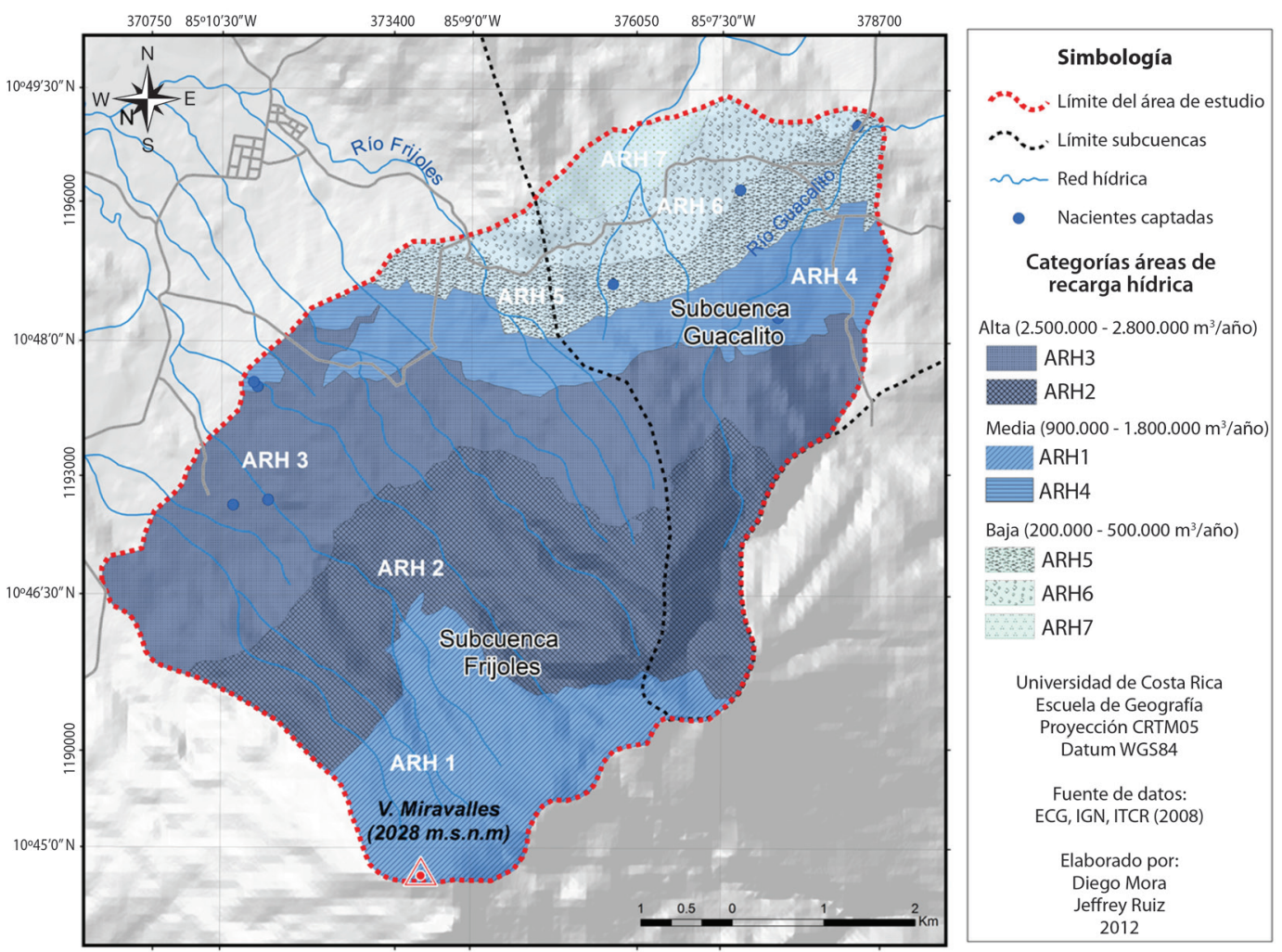

Figura 4. Categorización de las áreas de recarga en la parte media y alta de las subcuencas Frijoles y Guacalito, Upala.

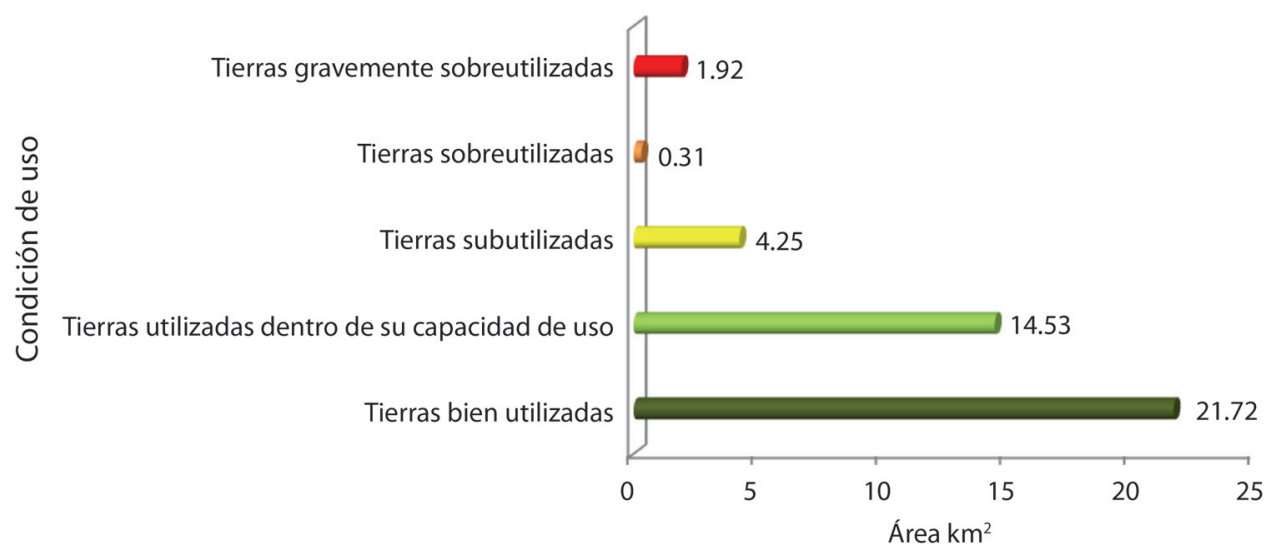

Figura 5. Condición de uso de la tierra en la parte media-alta de la subcuenca Frijoles y Guacalito, Upala. Elaborado por Mora y Ruiz, (2012).

del área. Esta categoría se ubica alrededor de los 1000 m.s.n.m. de ambas subcuencas, pero con una mayor presencia en Frijoles.

A continuación se presenta un cuadro con las acciones de manejo administrativo, legal y técnico sugeridas, seguido de la Figura 6 la cual muestra puntualmente las acciones de manejo sugeridas en las ARH de acuerdo con su categoría de recarga y su condición de uso de la tierra en el área de estudio:

\section{DISCUSIÓN}

Las ARH se caracterizan por tener un comportamiento descendente conforme disminuye 


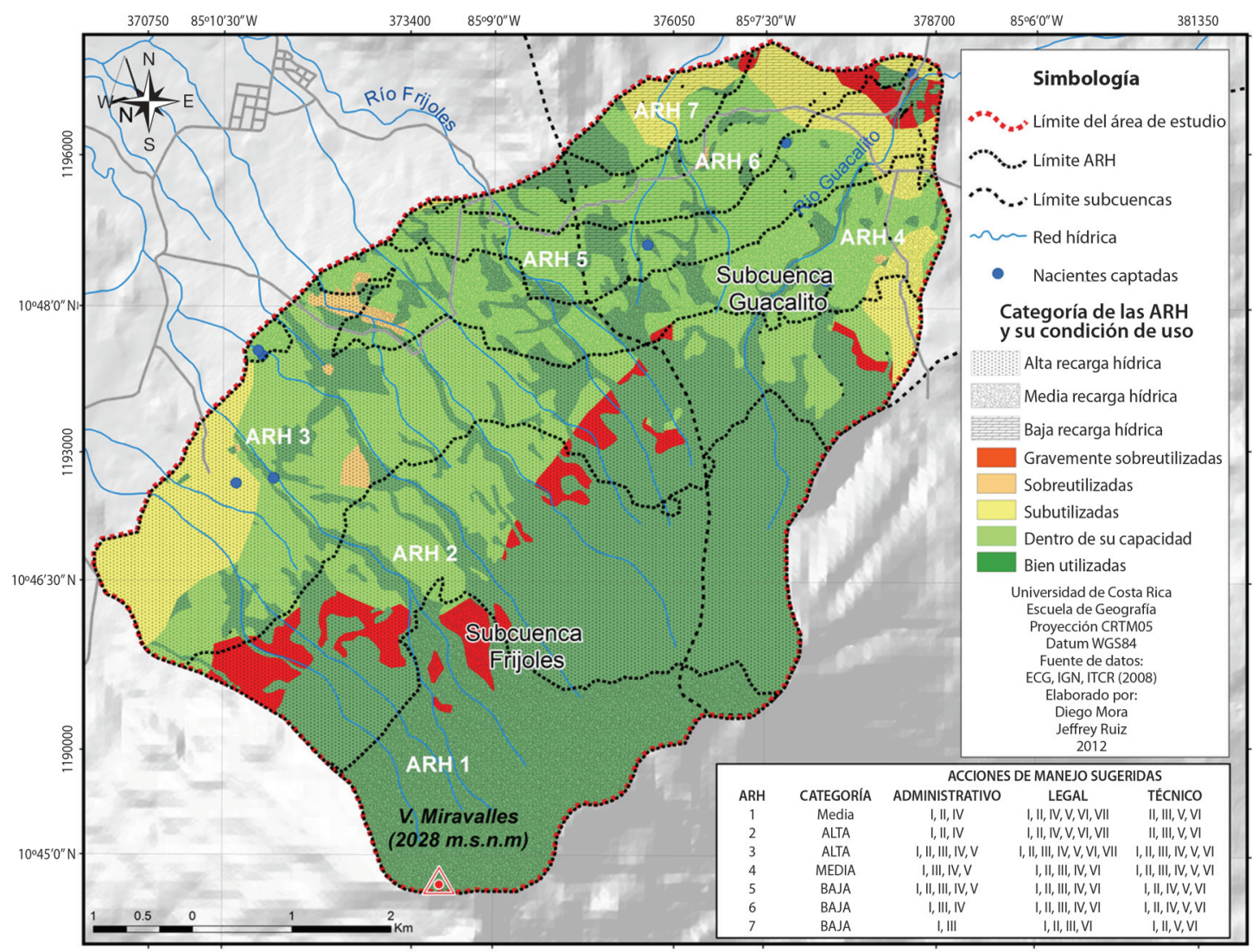

Figura 6. Acciones de manejo sugeridas en las ARH de acuerdo con su categoría de recarga y su condición de uso de la tierra en la parte media y alta de las subcuencas Frijoles y Guacalito, Upala.

la altitud, siguiendo un patrón similar al de las precipitaciones. En términos de recarga potencial, las ARH reciben un promedio de $4000 \mathrm{~mm} /$ año que se distribuye de manera irregular, siendo los sectores cercanos a la cumbre del volcán Miravalles los que reciben la mayor cantidad de precipitación, favoreciendo la recarga hídrica natural, principalmente las ARH localizadas en este sector (ARH2 y ARH3).

La distribución de las ARH está muy relacionada también con la evapotranspiración potencial y la cobertura vegetal, ya que ésta última disminuye la cantidad de agua disponible en zonas de menor altitud. Es por esta razón, que las ARH ubicadas en la parte alta (ARH1 y ARH2) presentan una mayor recarga potencial que aquellas ubicadas en los sectores medio y bajo (ARH5, ARH6 y ARH7). Lo mismo concluyeron Rodriguez y Perez (2014) demostrando que "en los bosques se da una mayor evapotranspiración, pero la capacidad de regulación y almacenamiento de humedad que presentan los suelos cubiertos de bosques, y la alta tasa de infiltración de los mismos, producen volúmenes de recarga superiores".

Las ARH de categoría alta se encuentran en zonas con fuertes pendientes pero cubiertas por bosques que reducen la escorrentía superficial. Es por este motivo que las ARH ubicadas a mayor altitud, presentan velocidades muy rápidas de infiltración a pesar de estar en zonas de fuertes pendientes. Si estas áreas estuvieran bajo usos diferentes al forestal es muy probable que la escorrentía aumentara y la infiltración disminuyera.

Las ARH ubicadas en zonas de pendientes fuertes, a pesar que cuentan con una mayor cobertura boscosa, mayor infiltración y una mayor recarga potencial; la escorrentía superficial 
TABLA 9

\section{Acciones de manejo administrativo, legal y técnico sugeridas}

Lineamiento Administrativo (A) I.
Acción de manejo sugerida

Monitoreo y vigilancia en las áreas de recarga determinadas, así como la supervisión de actividades cercanas a las fuentes de captación como la ganadería y la agricultura por medio de:

a. Control sobre los límites de las áreas de recarga hídrica

b. Tipos de uso de la tierra y prácticas agrícolas y ganaderas aplicadas

c. Cambios en la forma y características del acuífero ante eventos telúricos

d. Cambios en la permeabilidad del suelo

e. Estudios periódicos de calidad de agua

f. Medición de caudales y precipitación

II. Llevar a cabo una política de adquisición de terrenos en áreas de recarga hídrica gravemente sobreutilizadas. La municipalidad de Upala debe gestionar recursos para compra de terrenos en zonas inmediatas a las fuentes de captación de Upala.

II. Desarrollar campañas de divulgación entre la población local, donde se les explique la importancia de las áreas de recarga y su deterioro por condición de uso (ya sea prácticas agrícolas, ganaderas e industriales sin ningún control) que ponen en peligro el abastecimiento de agua. Asimismo, sensibilizar a la población sobre la necesidad de realizar actividades de protección ambiental y los beneficios que esta conlleva.

IV. Las autoridades locales deben controlar el desarrollo de cualquier actividad incompatible con la preservación del recurso hídrico, específicamente en las tierras sobreutilizadas y gravemente sobreutilizadas.

V. Las autoridades locales responsables del abastecimiento de agua, tienen que ser las encargadas de asegurar la calidad del agua evitando la contaminación de las mismas por prácticas agrícolas o ganaderas incorrectas.

Legal (L) I. Fortalecer la política ambiental municipal a través de la creación de un departamento de gestión ambiental que contemple la protección del recurso hídrico y demás recursos naturales del cantón.

II. Involucrar a todos los actores en la gestión y manejo del recurso hídrico en la zona, para llegar a un consenso sobre la distribución de funciones mediante una organización específica que coordine y vigile las actividades en las áreas de recarga.

III. Acatar las medidas de protección por la ley de aguas que exige un perímetro de $200 \mathrm{~m}$ a partir de los sitios de captación aguas arriba y la ley forestal, que aunque las medidas indicadas no se ajustan a la realidad, son de suma importancia.

IV. Aplicar medidas legales que aseguren el cumplimiento de las medidas previstas en las áreas de recarga hídrica que presentan condiciones de uso que vayan en contra de su protección, notificando a la población sobre restricciones en el uso de la tierra, contratos con los propietarios afectados y la inclusión de cláusulas específicas en los planes de desarrollo. La existencia de documentos oficiales garantiza que las medidas de protección continúen a través de los años.

V. Incentivar el pago por servicios ambientales como una herramienta específica para crear sistemas de manejo sostenible, principalmente en áreas de alta recarga que presenten condiciones de uso de tierras sobreutilizadas

VI. Aplicar un canon de agua donde las personas beneficiadas aporten una suma de dinero adicional con el fin de destinarlo a la protección de las áreas inmediatas de las fuentes de captación por medio de compra de árboles para reforestar cerca de ríos y manantiales con desprovista cobertura boscosa y alquiler de terreno para dueños de finca que limitan la producción hídrica

VII. Declarar con una categoría de manejo más restrictiva aquellas áreas de recarga que se encuentran dentro de la Zona Protectora Miravalles, para que la supervisión de éstas sea más rigurosa. 
Tabla 9 (Continuación)

Lineamiento

Técnico ( $\mathrm{T}$ )

\section{Acción de manejo sugerida}

I. Utilizar sistemas agroforestales en fincas individuales a través de la siembra de árboles o arbustos en áreas de cultivos o usos silvopastoriles, con especies nativas que absorban menor cantidad de agua, con el fin de disminuir la evapotranspiración, mejorar la capacidad de infiltración, porosidad, y permeabilidad y así facilitar el movimiento del agua en el suelo y subsuelo principalmente en las áreas de recarga hídrica críticas.

II. Realizar estudios de vulnerabilidad de las aguas subterráneas vinculadas a las condiciones hidrogeológicas locales asimismo, estudios hidrogeológicos que indiquen donde se encuentra el (los) acuífero(s) que alimenta los manantiales captados en la zona para establecer una zona forestal que proteja las áreas con mayor potencial de infiltración.

III. Realizar estudios técnicos más exhaustivos con base en la compactación y en el uso de la tierra, para realizar una zonificación de áreas críticas para el recurso hídrico, y de esta manera evitar la reducción de los caudales y la contaminación aguas abajo.

IV. Definir por medio de estudios técnicos, perímetros de protección en las zonas de captaciones, ya que generalmente sólo se toman en cuenta las medidas establecidas por la ley forestal y de aguas, sin embargo no son suficientes o no se adecuan a la realidad física donde están inmersas las fuentes de captación.

V. Realizar las siguientes prácticas para la conservación de suelos volcánicos en la zona.

a. No utilizar fertilizantes y agro tóxicos, según la recomendación técnica del MAG, ya que en la zona se dan velocidades de infiltración muy rápidas; y la utilización de agroquímicos contribuiría a que estos ingresen rápidamente en el subsuelo hasta alcanzar los mantos acuíferos que alimentan ríos, quebradas y manantiales.

b. Sembrar con base en las curvas de nivel.

c. Acequias y zanjas de infiltración para evitar la escorrentía.

d. Manejo de pastos y rotación de cultivos y de ganado para evitar la compactación.

e. Barreras vivas para evitar la erosión, ya que los suelos de la zona son de origen volcánico altamente erodables con usos intensivos de la tierra.

VI. Estimar la capacidad de carga de los suelos, ya que las sobrecargas inducen al pie de vaca, solifluxión y deslizamiento de los terrenos que ponen en riesgo la recarga de los acuíferos. Asimismo, en áreas de alta recarga hídrica donde existan tierras sobreutilizadas en pendientes mayores a 30\%, se hace necesario limitar las actividades ganaderas, ya que no son tierras aptas para soportar usos intensivos como se les están dando en la actualidad. limita el volumen de recarga hídrica anual en contraposición del ARH con una menor recarga potencial pero con un mayor área de influencia.

Las ARH localizadas en zonas con coberturas de bosques que poseen una estructura densa y con mayor cantidad de materia orgánica, son favorecidas con una mayor absorción de agua ya que la capa de humus que se forma en la base del suelo de un bosque protege al mismo de la compactación causada por la lluvia, regula la humedad y el flujo pausado del agua (Reynolds \& Thompson, 1988).

La estructura de los suelos también influye en la retención de agua en las ARH, la estructura de los suelos andisoles en el área de estudio, presentan horizontes subsuperficiales profundos que generan mayor espacio para almacenaje de agua. Igualmente, los resultados de textura mostraron, en su mayoría, texturas francas (franco arcillosos, franco limosos, franco arenosos) y con buen contenido de arcillas que por su naturaleza presentan una mayor capacidad de campo y a su vez retienen mayor cantidad de agua.

Los valores $\mathrm{Rp}$ en las ARH se pueden relacionar con la densidad aparente y compactación. En un estudio realizado por Henríquez Henríquez et al. (2011) se demostró que ambas variables presentan una relación directamente proporcional ya que a mayor compactación la densidad aparente era mayor y viceversa.

En áreas donde predominan coberturas boscosas, los muestreos realizados presentaron 
valores bajos de compactación mientras que en zonas con mayor presencia de pastos estos valores fueron más altos. Con base en estos resultados y analizando la distribución espacial, se infiere que las ARH ubicadas en el sector medio, al estar bajo predominio de pastos y pastos con árboles, presentan valores altos de compactación y de densidad aparente que limitan la recarga hídrica. Por otra parte, las áreas de recarga ubicadas en el sector alto (ARH1, ARH2 y ARH3), con mayor presencia de coberturas boscosas y con menor exposición a actividades agropecuarias intensivas, presentan valores de compactación y de densidad aparente menores que permiten una mayor infiltración. Justificando aún más la relación con la densidad aparente, los muestreos mostraron una tendencia que a menor densidad aparente del suelo mayor sería el agua útil que estaría disponible para infiltrar.

Las ARH ubicadas en el sector medio (ARH4, ARH5, ARH6 y ARH7) presentan en su mayoría usos de la tierra dedicados a cultivos y pastos para la ganadería, lo que genera que no se den valores altos de recarga potencial, ya que el uso de maquinaria y el pisoteo del ganado han aumentado la compactación disminuyendo a su vez las velocidades de infiltración y de recarga potencial. Este resultado es similar a las concluidas por investigaciones previas sobre prácticas de conservación de recursos naturales, por ejemplo, Faustino Manco et al. (2012) demostraron que en la cuenca del río Jesús María, la compactación por usos agropecuarios ha reducido y alterado la capacidad de infiltración de los suelos en un $52 \%$.

Las ARH, en general, presentan una distribución influenciada principalmente por el factor clima, ya que en zonas con mayor disponibilidad de agua hay un mayor potencial de recarga. De igual forma, el uso de la tierra influye en las variables geopedológicas como la densidad aparente, compactación y textura. Estas variables a su vez influyen directamente en la capacidad de infiltración y en la recarga hídrica potencial. La interacción de todas estos factores propician que en zonas de mayor altitud (cuenca hidrográfica alta) haya mayor potencial de recarga que en aquellas ubicadas en los sectores bajos de ambas subcuencas; sin embargo, la extensión de las ARH ubicadas en la parte media las hace de suma importancia en la recarga por volumen.

Así mismo, la capacidad de recarga potencial que tienen las ARH, es afectada por las condiciones de uso que se están originando en las mismas y que eventualmente podrían incidir en el equilibrio hídrico. Debido a esto, es indispensable definir algunos lineamientos de manejo legales, técnicos y administrativos a implementar en estas ARH que protejan y conserven el recurso hídrico a mediano y largo plazo, ya que conociendo su condición de uso, la intervención de las autoridades y población local es fundamental para el manejo del agua.

\section{CONCLUSIONES}

En la parte media y alta de las subcuencas Frijoles y Guacalito, existen importantes áreas con potencial de recarga hídrica, dado que el $51 \%$ del promedio de precipitación formará parte del agua subterránea; es decir, las condiciones biofísicas de la zona (pendientes, suelos de origen volcánico con texturas franco limosos, clima y vegetación en su mayoría boscosa), han contribuido a una constante recarga hídrica de los mantos acuíferos en ambas subcuencas, aún en meses de escasa precipitación.

Además, las subcuencas hidrográficas Frijoles y Guacalito están caracterizadas por condiciones biofísicas y fisiográficas similares como: extensa cobertura boscosa, precipitación y temperatura, suelos andisoles, una red fluvial desarrollada, formas similares, facilidad de concentraciones de lluvias intensas y cambios bruscos de pendiente; que ayudaron a determinar las áreas de recarga hídrica en el sitio. Estas condiciones y la intervención antrópica, han determinado dentro de las áreas de recarga hídrica, unidades de tierra con diferentes usos, aptitud y vulnerabilidad.

Por otro lado, la capacidad de infiltración fue mayor en bosque y menor en pastos, pastos con árboles y cultivos; no obstante, los resultados de la recarga potencial son más uniformes. Esto ocurre probablemente porque, la evapotranspiración real es mayor en bosque y menor en pastos, 
pastos con árboles y cultivos, de acuerdo con el balance hídrico. Estos valores de recarga potencial se obtuvieron en un tiempo y momento dado $\mathrm{y}$, en realidad, es el cambio de las propiedades físicas del suelo, la erosión, la compactación del suelo y el incremento de la escorrentía los que provocarán a futuro, una disminución de la recarga potencial en aquellos sitios que no están bajo cobertura boscosa.

La condición de uso en las áreas de recarga hídrica, muestra que la mayoría de las tierras están dentro de su capacidad de uso. Los conflictos de uso de la tierra, aún no ejercen la suficiente presión para provocar un deterioro inmediato en la zonas de recarga o en las fuentes de captación. Sin embargo, en las áreas de sobreuso y gravemente sobreutilizadas, el reto más importante es fortalecer el marco legal y, además, introducir incentivos para promover acciones (educación, pago por servicios ambientales, entre otros) que mejoren la toma de decisiones de cada ente encargado de velar por las subcuencas como unidad de gestión.

\section{RECOMENDACIONES}

Establecer una mayor vigilancia en las áreas con potencial de recarga hídrica mediante una estación meteorológica. Esto con el objetivo de medir y registrar el comportamiento de variables como precipitación y temperatura dentro del área de estudio. La información suministrada será relevante para la toma de decisiones ante la variabilidad climática y para futuras investigaciones que contribuyan con la protección del recurso hídrico.

Realizar estudios de capacidad de carga de los suelos, ya que por su origen volcánico presentan una alta porosidad lo que los hace más propensos a la compactación con usos muy intensivos de la tierra, lo que limitaría la infiltración básica.

Es necesarios realizar estudios complementarios que no sólo involucren el diagnóstico puntual de las fuentes de abastecimiento, sino también las áreas de recarga hídrica y las aguas residuales, ya que todas forman parte del sistema de abastecimiento de aguas de una comunidad.
Las autoridades encargadas del recurso hídrico en la zona, deben intervenir en las áreas de recarga hídrica baja, para propiciar un aumento de la recarga potencial por medio de la regeneración boscosa y otras actividades que propicien la infiltración, así mismo se debe velar que las condiciones favorables presentadas en áreas con un potencial alto de recarga sigan manteniéndose para garantizar la infiltración a futuro.

\section{REFERENCIAS}

Blanco Rojas, H. (2010). Área de recarga hídrica de la parte media-alta de las microcuencas Palo, Marín y San Rafaelito, San Carlos, Costa Rica: Cuadernos de investigación de la UNED. Recuperado de http://www. uned.ac.cr/investigacion/publicaciones/cuaderno1/ documents/06-Blanco-Areas.pdf.

Departamento de Agricultura de los Estados Unidos. (1999). Guía para la evaluación de la calidad y salud del suelo. Recuperado de http://soils.usda.gov/sqi/assessment/files/kitspanish.pdf

Escuela de Ciencias Geográficas. (2012). Capas de información cartográfica: Plan regulador para el cantón Upala. Universidad Nacional, Heredia, CR.

Faustino Manco, J., Gómez, M, Benegas Negri, L. Watler Reyes, W. J.\& Ney Ríos, J.(2012). Plan de Manejo de la cuenca del río Jesús María. Recuperado de http:// www.fonafifo.go.cr/documentacion/biblioteca/consultorias_investigaciones/ce_psa_006.pdf

Henríquez, C et al. (2011). Determinación de la resistencia a la penetración, al corte tangencial, densidad aparente y temperatura en un suelo cafetalero, Juan Viñas, Costa Rica. Agronomía costarricense. 35(1), 175-184. Recuperado de http://www.scielo.sa.cr/pdf/ac/v35n1/ a10v35n1.pdf

Henríquez Henríquez, C. (1999). Guía práctica para el estudio introductorio de los suelos con un enfoque agrícola. San José: EUNED.

Instituto Meteorológico Nacional. (2012). Información meteorológica referente a la estación Upala 69679. San José, Costa Rica.

Ley de Aguas, Ley 276. (1942). Asamblea Legislativa de Costa Rica. San José, Costa Rica.

Ley Forestal, Ley 7575. (1996). Asamblea Legislativa de Costa Rica. San José, Costa Rica. 
Ministerio de Ambiente y Energía. (2008). Política Hídrica Nacional. San José, Costa Rica:

Ministerio de Ambiente y Energía \& Ministerio de Agricultura y Ganadería. (2001). Decreto No. 29279-MINAE-MAG: Comisión Asesora sobre Degradación de Tierras (CADETI) Gaceta N. 30, 12 de febrero del 2001.

Mora Jiménez, L. D. y Ruiz Valverde, J. (2013). Determinación de áreas potenciales de recarga hídrica en la parte media y alta de las subcuencas Frijoles y Guacalito. (Tesis inédita de licenciatura). Universidad de Costa Rica, San Pedro, CR.
Noriega Arriaga, J. P. (2005). Determinación de las áreas principales de recarga hídrica natural en la microcuenca hidrográfica del río Sibacá, Chiniqué, Quiché. (Tesis inédita de licenciatura). Universidad de San Carlos de Guatemala, Ciudad de Guatemala.

Reynolds, E. y Thompson, F.(1988). Forest, climate and hydrology: Regional impact. United Nation University, Tokio.

Schosinsky Nevermann, G. (2006). Cálculo de la recarga potencial de acuíferos mediante un balance hídrico de suelos. Revista Geológica de América Central, 23, 43-55. Recuperado de http://www.geologia.ucr.ac.cr/ revista/to_pdf/revista/34-35/02-schosinsky1.pdf 